\title{
Pavel Tomasta
}

On decompositions of complete $k$-uniform hypergraphs

Czechoslovak Mathematical Journal, Vol. 28 (1978), No. 1, 120-126

Persistent URL: http://dml.cz/dmlcz/101518

\section{Terms of use:}

(C) Institute of Mathematics AS CR, 1978

Institute of Mathematics of the Czech Academy of Sciences provides access to digitized documents strictly for personal use. Each copy of any part of this document must contain these Terms of use.

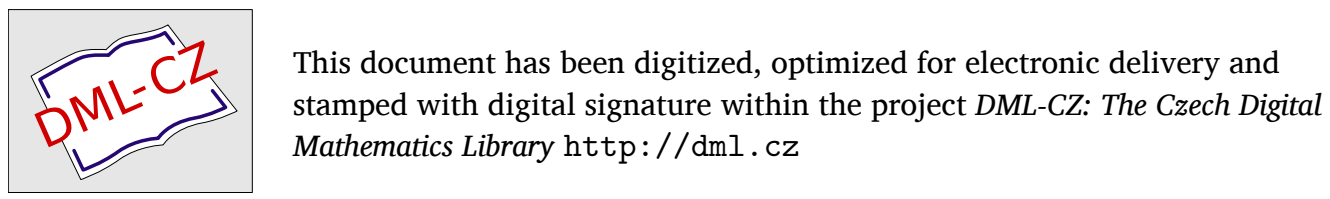




\title{
ON DECOMPOSITIONS OF COMPLETE $k$-UNIFORM HYPERGRAPHS
}

\author{
Pavel Tomasta, Bratislava
}

(Received January 19, 1976)

\section{INTRODUCTION}

The problem of decomposing graphs or hypergraphs into factors with given properties occurs in many modifications. One of these is to determine some conditions for the existence of a decomposition of a complete $k$-uniform hypergraph into $m$ factors with given diameters $d_{1}, d_{2}, \ldots, d_{m}$. Our aim is to determine the smallest cardinal number $F^{k}\left(d_{1}, d_{2}, \ldots, d_{m}\right)=t$ (if such a cardinal number exists) such that a complete $k$-uniform hypergraph $K_{t}^{k}$ on $t$ vertices can be decomposed into $m$ factors with diameters $d_{1}, d_{2}, \ldots, d_{m}$ and (if it is possible) to find the decomposition required by a constructive method.

In [1] it was proved that $F^{2}\left(d_{1}, d_{2}, \ldots, d_{m}\right) \leqq m d-m$ for $d \geqq 3$ and $m \geqq 3$, where $d=\max d_{i}$. Relatively more complicated it was to find a decomposition into factors with diameters equal to two. J. Bosák, P. ERDös, A. Rosa in [2] proved that

$$
4 m-1 \leqq F_{m}^{2}(2) \leqq\left(\begin{array}{l}
6 m-2 \\
2 m-2
\end{array}\right) .
$$

Later on, J. Bosák proved in [3] an inequality which is the best one known till now:

$$
6 m-52 \leqq F_{m}^{2}(2) \leqq 6 m \text {. }
$$

The papers [9], [11], [10] investigate the problem of decomposing a complete digraph into factors with given diameters.

D. Palumbíny in [5], [6] studied the problem of decomposing a complete graph into factors with equal diameters. He proved in [5] that $F_{m}^{2}(d)=2 m$ for $m \geqq 2$ and $3 \leqq d \leqq 2 m-1$. Even though his aim was not to find a decomposition into isomorphic factors, $m$ factors with diameter equal to $d$ of his decomposition of the complete graph with $2 m$ vertices are isomorphic for $d$ odd. The study of the decompositions of complete graphs into isomorphic factors with given diameters was initiated in [4], where the decomposition of a complete graph into three isomorphic factors with a given diameter $d \geqq 2$ is considered. 
In [7] the problem of decomposing a complete $k$-uniform hypergraph $(k \geqq 2)$ into isomorphic factors with a given diameter was systematically studied. It seems that this is the shortest way to solve the problem of the existence of the cardinal number $F^{k}\left(d_{1}, d_{2}, \ldots, d_{m}\right)$.

We shall give in this paper an upper bound for the number $F^{k}\left(d_{1}, d_{2}, \ldots, d_{m}\right)$ where $3 \leqq k<m$ and $2 \leqq d_{1} \leqq d_{2} \leqq \ldots \leqq d_{m}$. The case when at least one of the diameters is equal to one was solved in [8].

First we give some notations and definitions. A hypergraph is an ordered pair of sets $G=(V, H)$ wheie $H \subset P(V)$ (the potency of $V)$. Let $k$ be a positive integer. A hypergraph $G$ is said to be a $k$-uniform hypergraph if for each $h \in H$ we have $|h|=k$. For $k=2$ we obtain graphs. If the set $H$ contains all the $k$-element subsets of $V$ then $G$ is said to be a complete k-uniform hypergraph and we denote $G$ by $K_{n}^{k}$ where $n=|V|$. The distance $d(x, y)$ of two vertices $x$ and $y$ is the length of the shortest path joining them. The diameter of a hypergraph is defined by $d=$. $=\sup _{x, y \in V} d(x, y)$.

A factor of $G$ is such a subhypergraph of $G$ which contains all vertices of $G$. Let $F^{k}\left(d_{1}, d_{2}, \ldots, d_{m}\right)=t$ be the smallest integer (if it exists) such that the hypergraph $K_{t}^{k}$ is decomposable into $m$ factors with diameters $d_{1}, d_{2}, \ldots, d_{m}$. If $d_{1}=\ldots=d_{m}=d$ we put $F_{m}^{k}(d)=F^{k}\left(d_{1}, d_{2}, \ldots, d_{m}\right)$. We shall say that $G_{1}=\left(V_{1}, H_{1}\right)$ and $G_{2}=$ $=\left(V_{2}, H_{2}\right)$ are isomorphic if there exists a bijection $f: V_{1} \rightarrow V_{2}$ such that $h \in H_{1}$ if and only if $f(h) \in \mathrm{H}_{2}$.

Let $G_{m}^{k}(d)=t$ be the smallest cardinal number such that $K^{k}$ can be decomposed into $m$ isomorphic factors with the diameter $d$.

Definition 1. Let $G$ be an arbitrary group of automorphisms of the hypergraph $K_{n}^{k}$ and let there exist a surjection $h: G \rightarrow R$, where $R$ is a decomposition of the hypergraph $K_{n}^{k}$ into isomorphic factors, with the following property:

$$
x(h(y))=h(x y) \text { for every } x, y \in G .
$$

Then we shall say that $R$ is a decomposition of $K_{n}^{k}$ by the group $G$. If the mapping $h$ is a bijection then we shall say that $R$ is a simple decomposition of $K_{n}^{k}$ by $G$. The factor $h(x)$ will be denoted by $G_{x}$.

In our further considerations we shall need the following propositions.

Proposition 1. Let $H$ be an Abelian group of a finite order $m>1$ and let $k \geqq 3$ be a natural number such that $(m, k !)=1$. Then the following two statements are equivalent:

1. There exists a group $H_{1} \cong H$ such that the hypergraph $K_{n}^{k}$ has a simple decomposition by the group $H_{1}$. 
2. $m$ divides $\left(\begin{array}{l}n \\ k\end{array}\right)$ and divides just one of the numbers $n, n-1, \ldots, n-k+1$.

This statement was proved in [7]. It was also remarked there that a weaker condition is sufficient for the existence of a simple decomposition of $K_{n}^{k}$, namely $(m, k)=1$. We use this fact in the sequel.

Proposition 2. Let $F^{k}\left(d_{1}, d_{2}, \ldots, d_{m}\right)$ exist. Then the complete hypergraph $K_{k}^{N}$ can be decomposed into $m$ factors with diameters $d_{1}, d_{2}, \ldots, d_{m}$ if and only if $N \geqq F^{k}\left(d_{1}, d_{2}, \ldots, d_{m}\right)$.

Proposition 3. Let $3 \leqq k<m,(m, k)=1, d \geqq 2$ be integers. Then $G_{m}^{k}(d)$ exists and

$$
G_{m}^{k}(d) \leqq m[(d-2)(k-1)+] \quad \text { if } \quad d \geqq 3, \quad G_{m}^{k}(2) \leqq 2 m .
$$

Proposition 2 was proved in [8] and Proposition 3 in [7]. Now we are able to prove

Theorem 1. Let $3 \leqq k<m,(m, k)=1,2 \leqq d_{1} \leqq d_{2} \leqq \ldots \leqq d_{m}$ be integers. Then $F^{k}\left(d_{1}, d_{2}, \ldots, d_{m}\right)$ exists and

$$
\begin{aligned}
& F^{k}\left(d_{1}, d_{2}, \ldots, d_{m}\right) \leqq m\left[\left(d_{m}-2\right)(k-1)+1\right] \quad \text { if } d_{m} \geqq 3, \\
& F^{k}\left(d_{1}, d_{2}, \ldots, d_{m}\right) \leqq 2 m \text { if } d_{m}=2 .
\end{aligned}
$$

Proof. I. Assume $d_{m} \geqq 3$ and put $n=m t$ where $t=\left(d_{m}-2\right)(k-1)+1$. Denote the vertices of $K_{n}^{k}$ by $i_{j}, 1 \leqq i \leqq m, 1 \leqq j \leqq t$. Obviously $m$ divides $\left(\begin{array}{l}n \\ k\end{array}\right)$. Moreover, $m$ divides $n$. Because $(m, k)=1$, the sufficient condition for the existence of a simple decomposition of $K_{n}^{k}$ by a cyclic group $H$ of order $m$ generated by a permutation $\beta=\left(1_{1}, 2_{1}, \ldots, m_{1}\right)\left(1_{2}, 2_{2}, \ldots, m_{2}\right) \ldots\left(1_{t}, 2_{t}, \ldots, m_{t}\right)$ is satisfied. In the proof of Proposition 3 a special simple decomposition $R=\left\{G_{\alpha} / \alpha \in H\right\}$ of $K_{n}^{k}$ by the group $H$ was constructed, the factors of which have the diameters equal to $d$.

The factors $G_{\alpha}$ are constructed as follows:

1. The factor $G_{0}$ corresponding to zero of $H$ contains a path of length $d-2$ formed by the edges

$$
h_{i}=\left\{1_{t_{i}}, 1_{t_{i}+1}, \ldots, 1_{t_{i+1}}\right\} \quad \text { where } \quad t_{i}=1+(i-1)(k-1), 1 \leqq i \leqq d-2
$$

and the edge $f=\left\{1_{1}, 2_{1}, \ldots, k_{1}\right\}$.

2. $\left\{B \cup\left\{2_{j}\right\}\right\} \in G_{0}$ for any $(k-1)$-tuple $B \subset A_{i}=\left\{2_{i}, 3_{i}, \ldots, m_{i}\right\}, i \neq j, i, j=$ $=1,2, \ldots, t$.

3. $G_{\alpha}=\alpha\left(G_{0}\right)$ for every $\alpha \in H$.

Obviously the diameter of $G_{\alpha}$ is equal to $d$.

4. All the remaining edges are divided into factors preserving the diameters. 
Now we shall modify this decomposition $R$ of the hypergraph $K_{n}^{k}$ in order to obtain the required diameters of the factors. Put $S=\left\{f_{i}=\left\{1_{i}, 2_{i}, \ldots, k_{i}\right\} / i=2,3, \ldots, t\right\}$. Choose the edges $\alpha(S)$ for every $\alpha \in H$ from the factors of the decomposition $R$. It is evident that the diameters of the factors remain the same as before. Let $p\left(d_{i}\right)$ denote the number of the members of the set $D=\left\{d_{1}, d_{2}, \ldots, d_{m}\right\}$ which are equal to $d_{i}$.

(a) Assume $d_{1}=2$. Then insert the edges $f_{i}, i=2,3, \ldots, t$ into the factor $G_{0}$ and the edges $\beta^{j-1}\left(f_{i}\right)$ into the factor $G_{\beta^{j-1}}$ where $2 \leqq j \leqq p(2)$.

(b) Assume $d_{1}>2$. If $d_{1}=d_{m}$ then all the diameters are the same and equal to $d_{m}$. However, by Proposition 3 the number $G_{m}^{k}\left(d_{m}\right)$ exists. Since $F_{m}^{k}\left(d_{m}\right) \leqq G_{m}^{k}\left(d_{m}\right)$ the proof is completed. So we can assume $d_{1}<d_{m}$. Then there exists the greatest index $x$ such that $d_{G_{0}}\left(1_{x}, 1_{t}\right)=d_{1}-2$ (the index $G_{0}$ denotes the distance taken in $\left.G_{0}\right)$. Insert the edges $f_{i}, i=2,3, \ldots, x$ into the factor $G_{0}$ and the edges $\beta^{j-1}\left(f_{i}\right)$ into the factors $G_{\beta^{j-1}}, 2 \leqq j \leqq p\left(d_{1}\right), 2 \leqq i \leqq x$.

Now consider an arbitrary $d_{r} \in D, d_{1}<d_{r}<d_{m}$. If such $d_{r}$ does not exist the required decomposition is achieved, because the factors $G_{\beta s}$ where $p\left(d_{1}\right) \leqq s<m$ have the diameter equal to $d_{m}$ and the remaining edges of the sets $\alpha(S)$ can be put back into the same factors from which they were chosen.

Let such a $d_{r}$ exist. Denote by $r^{0}$ the smallest number such that $d_{r^{0}}=d_{r}$. We can assume without loss of generality that $j \leqq r$ for every $d_{j}=d_{r}$. Since $d_{r}<d_{m}$, there exists the greatest index $x$ such that

$$
d_{G^{\beta}}{ }^{r 0-1}\left(\left(r^{0}\right)_{x},\left(r^{0}\right)_{t}\right)=d_{r}-2 .
$$

Then let $\beta^{s}\left(f_{i}\right) \in G_{\beta^{s}}$ where $r^{0}-1 \leqq s<r$. Insert all the other edges from the sets $\beta^{s}(S), r^{0}-1 \leqq s<r$ into $G_{\beta^{0}}-1$. It is evident that the diameter of the factors $G_{\beta^{s}}$ constructed in this way is equal to $d_{r}$.

This construction can be applied to every diameter $d_{q}<d_{m}$. Denote by $m^{0}$ the smallest number such that $d_{m^{0}}=d_{m}$ and insert the edges $\beta^{s}(S), m^{0}-1 \leqq s<m$ into the same factors from which they were chosen.

It can be easily verified that $\left\{G_{\alpha} / \alpha \in H\right\}$ is the required decomposition of the hypergraph $K_{n}^{k}$ into factors with diameters $d_{1}, d_{2}, \ldots, d_{m}$.

II. Assume $d_{m}=2$. Then all the diameters are equal to 2. By Proposition 3 the number $G_{m}^{k}(2)$ exists and since $F_{m}^{k}(2) \leqq G_{m}^{k}(2) \leqq 2 m$ the proof is complete.

In the following theorem the existence of the number $F^{k}\left(d_{1}, d_{2}, \ldots, d_{m}\right)$ in the case $(m, k)>1$ will be investigated. The next proposition proved in [7] will be very useful for the purpose.

Proposition 4. Let $3 \leqq k<m,(m, k)>1, d \geqq 2$ be integers. Then $G_{m}^{k}(d)$ exists and

$$
\begin{aligned}
& G_{m}^{k}(d) \leqq k m[(d-2)(k-1)+1] \quad \text { if } \quad d \geqq 3, \\
& G_{m}^{k}(2) \leqq 2 m k .
\end{aligned}
$$


Theorem 2. Let $3 \leqq k<m,(m, k)>1,2 \leqq d_{1} \leqq d_{2} \leqq \ldots \leqq d_{m}$ be integers. Then $F^{k}\left(d_{1}, d_{2}, \ldots, d_{m}\right)$ exists and

$$
\begin{aligned}
& F^{k}\left(d_{1}, d_{2}, \ldots, d_{m}\right) \leqq m k\left[\left(d_{m}-2\right)(k-1)+1\right] \text { if } d_{m} \geqq 3, \\
& F^{k}\left(d_{1}, d_{2}, \ldots, d_{m}\right) \leqq 2 m k \text { if } d_{m}=2 .
\end{aligned}
$$

Proof. I. Assume $d_{m} \geqq 3$. Put $n=k m t$ where $t=\left(d_{m}-2\right)(k-1)+1$. Denote the vertices of the hypergraph $K_{n}^{k}$ by $i_{j}, 1 \leqq i \leqq k m, 1 \leqq j \leqq t$. Let $H$ be the group generated by the permutation $\beta=\left(1_{1}, 2_{1}, \ldots,(\mathrm{km})_{1}\right)\left(1_{2}, 2_{2}, \ldots,(\mathrm{km})_{2}\right) \ldots$ $\ldots\left(1_{t}, 2_{t}, \ldots,(\mathrm{km})_{t}\right)$. In the proof of Proposition 4 , a decomposition $R$ of $K_{n}^{k}$ by the cyclic group $H$ of order $\mathrm{km}$ into $m$ isomorphic factors with diameter $d_{m}$ was found.

The factors $G_{\alpha}$ were constructed as follows:

1. $h_{j}=\left\{m_{j},(2 m)_{j}, \ldots,(k m)_{j}\right\} \in G_{0}$ for $1 \leqq j \leqq t$, where $G_{0}$ is the factor corresponding to the zero element of $H$.

2. The factor $G_{0}$ contains a path of length $d_{m}-2$ :

Put $f_{s}=\left\{m_{t_{s}},(2 m)_{t_{s}+1}, \ldots,(k m)_{t_{s+1}}\right\}, t_{s}=1+s(k-1), 0 \leqq s<d_{m}-2$. Then $\beta^{m r}\left(f_{s}\right) \in G_{0}$ for every $1 \leqq r \leqq k, 0 \leqq s<d_{m}-2$.

3. Put $A_{j}=\left\{i_{j} \mid 1 \leqq i \leqq k m\right\}-h_{j}$ and take an arbitrary $(k-1)$-tuple $B \subset A_{j}$. Then $\beta^{m r}\left(B \cup(m+1)_{i}\right) \in G_{0}$ for every $i \neq j, i, j=1,2, \ldots, t, 1 \leqq r \leqq k$. The edge $g \subset\left\{1_{1}, 2_{1}, \ldots,(\mathrm{km})_{1}\right\}$ which contains the vertices $m_{1},(m+1)_{1}$ and no other vertices of $h_{1}$ is also inserted into the factor $G_{0}$, together with its images $\beta^{m r}(g)$, $1 \leqq r \leqq k$.

Obviously the diameter of $G_{0}$ is equal to $d_{m}$. If we put $G_{\beta^{i}}=\beta^{i}\left(G_{0}\right)$ for every $0 \leqq i<m$, then the factors $G_{\beta^{i}}$ have the diameters equal to $d_{m}$.

All the remaining edges are divided into factors preserving the diameters.

Now we shall modify this decomposition $R$ with the aim to obtain the required diameters of the factors. Put

$$
S=\left\{e_{i}=\left\{m_{i},(m+1)_{i}, \ldots,(m+k-1)_{i}\right\} / 2 \leqq i \leqq t\right\} .
$$

Choose the edges $\alpha(S)$ for every $\alpha \in H$ from the factors of the decomposition $R$. It is evident that the diameters of the factors remain the same as before. Let $p\left(d_{i}\right)$ denote the number of members of the set $D=\left\{d_{1}, d_{2}, \ldots, d_{m}\right\}$ which are equal to $d_{i}$.

(a) Suppose $d_{1}=1$. Then insert the edges $\beta^{m r}\left(e_{i}\right)$ into the factor $G_{0}$ and the edges $\beta^{r(j-1)}\left(e_{i}\right)$ into the factor $G_{\beta^{j-1}}$, where $1 \leqq i \leqq t, 1 \leqq r \leqq k, 2 \leqq j \leqq p(2)$.

(b) Suppose $d_{1}>2$. If $d_{1}=d_{m}$ then all the diameters are the same and equal to $d_{m}$. However, by Proposition 4 the number $G_{m}^{k}\left(d_{m}\right)$ exists. Since $F_{m}^{k}\left(d_{m}\right) \leqq G_{m}^{k}\left(d_{m}\right)$ the proof is completed. So it can be supposed $d_{1}<d_{m}$. Then there exists the greatest index $x$ such that $d_{G_{0}}\left(m_{x}, m_{t}\right)=d_{1}-2$. Insert the edges $\beta^{m r}\left(e_{i}\right)$ into the factor $G_{0}$ and the edges $\beta^{r(j-1)}\left(e_{i}\right)$ into the factors $G_{\beta^{j-1}}$ where $2 \leqq j \leqq p\left(d_{1}\right), 2 \leqq i \leqq x$, $1 \leqq r \leqq k$. 
Now consider an arbitrary $d_{r} \in D, d_{1}<d_{r}<d_{m}$. If such $d_{r}$ does not exist the required decomposition is achieved, because the factors $G_{\beta^{s}}, p\left(d_{1}\right) \leqq s<m$ have the diameters equal to $d_{m}$ and the remaining edges of the sets $\alpha(S)$ can be put back into the same factors from which they were chosen.

Let such a $d_{r}$ exist. It can be supposed without loss of generality that $j \leqq r$ for every $d_{j}=d_{r}$. Denote by $r^{0}$ the smallest number such that $d_{r^{0}}=d_{r}$. Since $d_{r}<d_{m}$ there exists the greatest index $x$ such that

$$
d_{G^{\beta^{r 0-1}}}\left(\left(m+r^{0}\right)_{x},\left(m+r^{0}\right)_{t}\right)=d_{r}-2 .
$$

Then let $\beta^{m s}\left(e_{i}\right) \in G_{\beta^{s}}$ where $r^{0}-1 \leqq s<r, 2 \leqq i \leqq x$. Insert all the other edges from the sets $\beta^{s}(S), r^{0}-1 \leqq s<r$ into the factor, say, $G_{\beta^{0}-1}$. It is evident that the diameters of the factors $G_{\beta^{s}}, r^{0}-1 \leqq s<r$ constructed in this way are equal to $d_{r}$.

This construction can be applied to every $d_{q}<d_{m}$. Denote by $m^{0}$ the smallest number such that $d_{m^{0}}=d_{m}$ and insert the edges $\beta^{s}(S), m^{0}-1 \leqq s<m$ into the same factors from which they were chosen.

It can be easily verified that $\left\{G_{\alpha} / \alpha \in H\right\}$ is the required decomposition of the hypergraph $K_{n}^{k}$ into factors with diameters $d_{1}, d_{2}, \ldots, d_{m}$.

II. Suppose $d_{m}=2$. Then all the diameters are equal to 2. By Proposition 4 the number $G_{m}^{k}(2)$ exists and since $F_{m}^{k}(2) \leqq G_{m}^{k}(2) \leqq 2 \mathrm{~km}$ the proof is complete.

The preceding results yield the following theorem.

Theorem 3. Let $3 \leqq k<m, 2 \leqq d_{1} \leqq d_{2} \leqq \ldots \leqq d_{m}, d_{m} \geqq 3$, be integers.

If $(m, k)=1$ and $n \geqq m\left[\left(d_{m}-2\right)(k-1)+1\right]$ then $K_{n}^{k}$ can be decomposed into $m$ factors with diameters $d_{1}, d_{2}, \ldots, d_{m}$.

If $(m, k)>1$ and $n \geqq k m\left[\left(d_{m}-2\right)(k-1)+1\right]$ then $K_{n}^{k}$ can be also decomposed into $m$ factors with diameters $d_{1}, d_{2}, \ldots, d_{m}$.

Proof. Applying Proposition 2 to Theorems 1 and 2 we obtain the above statement.

\section{References}

[1] J. Bosák, A. Rosa, S̆. Znám: On decompositions of complete graphs into factors with given diameter, Theory of graphs, Proc. Colloq. Tihany 1966, Publ. House Hungarian Acad. Sci. Budapest 1968, 37-56.

[2] J. Bosák, P. Erdös, A. Rosa: Dəcompositions of complete graphs into factors with diameter two, Mat. čes. 21 (1971), 14-28.

[3] J. Bosák: Disjoint factors of diameter two in complete graphs, J. Combinatorial Theory B 16 (1974), 57-63.

[4] A. Kotzig, A. Rosa: Dicomposition of complete graphs into isomorphic factors with a given diameter, Bull. London Math. Soc., 7 (1975), 51-57.

[5] D. Palumbiny: On decompositions of complete graphs into factors with equal diameters, Boll. Unione Mat. Ital., 7 (1973), 420-428.

[6] D. Palumbiny: On a certain type of decompositions of complete graphs into factors with equal diameters, Mat. čas., 22 (1972), 235-242. 
[7] P. Tomasta: Decompositions of graphs and hypergraphs into isomorphic factors with a given diameter, Czechoslovak Math. J., 27 (102) 1977, 598-608.

[8] P. Tomasta: Decompositions of complete $k$-uniform hypergraphs into factors with given diameters, Comment. Math. Univ. Carolinae, 17 (1976), 377-392.

[9] E. Tomova: On the decomposition of the complete directed graph into factors with given diameters, Mat. čas., 20 (1970), 257-261.

[10] Ś. Znám: Decompositions of the complete directed graphs into factors with given diameters, Combinat. Structures and their applications, Proc. Calgary Internat. Confer., 1969, Gordon and Breach, N.Y., (1970), 489-490.

[11] $\breve{S}$. Znám: Dzcomposition of complete directed graph into two factors with given diameters, Mat. čas., 20 (1970), 254-- 256.

Author's address: 88625 Bratislava, Obrancov mieru 49, ČSSR (Matematický ústav SAV). 